\title{
Antenna Selection and Detection Performance on Correlation Based Detection Systems
}

\author{
F. YAVUZ ILGIN
}

\begin{abstract}
The increase data size transmitted in digital communication systems necessitates the use of high-capacity communication channels for the transmission of these data. To increase the channel capacity, MIMO systems are widely used. Antenna selection in MIMO systems is very important for efficient use of communication sources. Cognitive Radio systems generally aim to increase spectrum efficiency by using MIMO systems. Therefore, Cognitive Radio must find empty spectrum regions with spectrum detection methods. Spectrum detection performance is related to the antenna numbers in MIMO systems. Therefore, this study examines the effect of antenna numbers on detection performance in correlation-based detection method. Thanks to this study, the relationship between channel capacity, detection performance and antenna number for MIMO systems was investigated.
\end{abstract}

Index Terms-Antenna Selection, Cognitive Radio, Correlation based Spectrum Sensing, MIMO systems, Spectrum Sensing .

\section{INTRODUCTION}

It is known that the amount of data transmitted in wireless communication systems is gradually increasing[1]. To transmit large amounts of data (for example DTV, 5G communication signal), more channel capacity is required[2]. Multiple Input Multiple Output (MIMO) wireless communication systems provide a significant improvement in system efficiency as they have multiple receiver-transceiver antennas[3]. One of the biggest advantages of MIMO systems is that their channel capacities are much higher than Single Input Single Output (SISO) systems[4]. However, MIMO systems contain amplifiers, analog-to-digital converters, mixers that are very expensive and complex. Despite these known disadvantages, MIMO systems are widely preferred due to their advantages. In MIMO systems, it is important to use the least antenna (both transmitter and receiver) to meet the system requirements[5].

In some communication applications, the use of multiple antennas is a necessity, not just because of the contribution

FATIH YAVUZ ILGIN, is with Department of Electrical Electronical Engineering Erzincan Binali YILDIRIM University, Erzincan, Turkey,(email: (fyilgin@erzincan.edu.tr).

iD https://orcid.org/0000-0002-7449-4811

Manuscript received August 28, 2020; accepted September 22, 2020. DOI: $10.17694 /$ bajece. 787485 that MIMO systems can provide[6]. One of these applications is Cognitive Radio (CR) applications. The use of multiple antennas in multi-antenna CR systems is indispensable for some spectrum sensing models. For example, in eigenvalue and covariance based detection models, it is necessary to use multi-antenna in both transmitter and receiver[7]. However, there is no such requirement in the energy based sensing model. Basically, spectrum detection applications in CR systems are divided into classes such as single user-single antenna, single user-multi antenna or multi-user single antenna. Which method will be chosen to detect spectrum should be selected depending on some hardware requirements (number of users, antenna numbers) and communication environment. The main aim in spectrum sensing applications is to realize the most successful detection in the presence of the highest noise. Nevertheless, this process is not easy due to noise, fading and other disruptive effects in the wireless communication environment[8]. For this reason, various methods are proposed to ensure the most successful detection with the least user or antenna by using collaboration detection or some grouping algorithms[9]. The correlation-based detection method is very advantageous in terms of both computational cost and detection performance. In addition, the detection method used in this study detects as blind. That is, it does not need any prior knowledge for detection(for example: noise variance, modulation scheme).

Increasing the antenna numbers in spectrum sensing applications may increase detection performance, but increasing the antenna numbers is difficult in spectrum sensing applications. Because the antennas must be far enough from each other to not correlate with each other. On the other hand, the increase in the antenna numbers both increases the channel capacity and positively affects the detection performance.

Therefore, in this study, channel capacity-sensing performances are evaluated for correlation-based spectrum sensing in MIMO-OFDM systems. Thus firstly, theoretical information is given by channel capacity and correlation based spectrum sensing method for MIMO systems. Following, the relationship between antenna number and sensing performance in MIMO -OFDM based spectrum sensing method was investigated.

The organization of the study is as follows. Section 2 describes the antenna selection and channel capacity for MIMO systems. Cognitive radio and correlation-based spectrum detection is explained in chapter 3 and necessary 
theoretical information is given. The results of the simulation studies made in section 4 are given. Chapter 5 is reserved for discussion and conclusion.

Some of the notation we use is as follows: superscript $(.)^{T}$ and $\mathbf{I}_{L}$ stands for transpose and the identity matrix of order $\boldsymbol{L}$ respectively. And $E($.$) denotes expectation operation.$

\section{Channel Capacity MiMO systems}

Suppose there is $N_{t} x N_{r}$ MIMO communication system, where $N_{t}$ and $N_{r}$ indicate the number of transmit and receive antennas, respectively. With this communication system, the maximum throughput achieved by transmitting $\min \left(N_{t} x N_{r}\right)$ number of parallel data stream, is given by[10]

$$
B=\log _{2} \operatorname{det}\left(\boldsymbol{I}_{N_{r}}+\frac{S}{\eta \mathrm{N}_{\mathrm{t}}} \boldsymbol{H}^{T}\right) \text { bps } / \mathrm{Hz}
$$

where, $\boldsymbol{I}_{N_{r}} \boldsymbol{\epsilon} C^{N_{r} \mathrm{x} \mathrm{N}_{\mathrm{r}}}$ and $\boldsymbol{H} \boldsymbol{\epsilon} C^{\mathrm{N}_{\mathrm{t}} \mathrm{x} \mathrm{N}_{\mathrm{r}}}$ are the identity matrix and channel coefficient matrix, respectively. $\eta$ and is the power spectral density of additive Gaussian noise with $\eta \sim N\left(0, \sigma_{\eta}^{2}\right)$. In addition $S$ denotes the transmitted signal power.

Antenna selection technique aims to reduce the number of transmitting antennas without changing the system efficiency by using the channel information on the receiver. Optimum throughput is acquired through selection of best set of $K$ antennas out of $C_{M}$ combinations. Where $M$ defines the number of transmit antennas to be used for transmission. Thus, the relationship between optimum system efficiency and antenna number is defined by Channel State Information (CSI) as follows[11].

$B_{M}^{o p t}=\log _{2} \operatorname{det}\left(\boldsymbol{I}_{N_{r}}+\frac{S}{\eta N_{t}} \boldsymbol{H}_{\gamma}^{o p t}\left(\boldsymbol{H}_{\gamma}^{o p t}\right)^{T}\right)$ bpsHz

where, $B_{M}^{o p t}$ is the channel capacity for optimally selected antenna number. The traditional MIMO system is given in Fig 1.

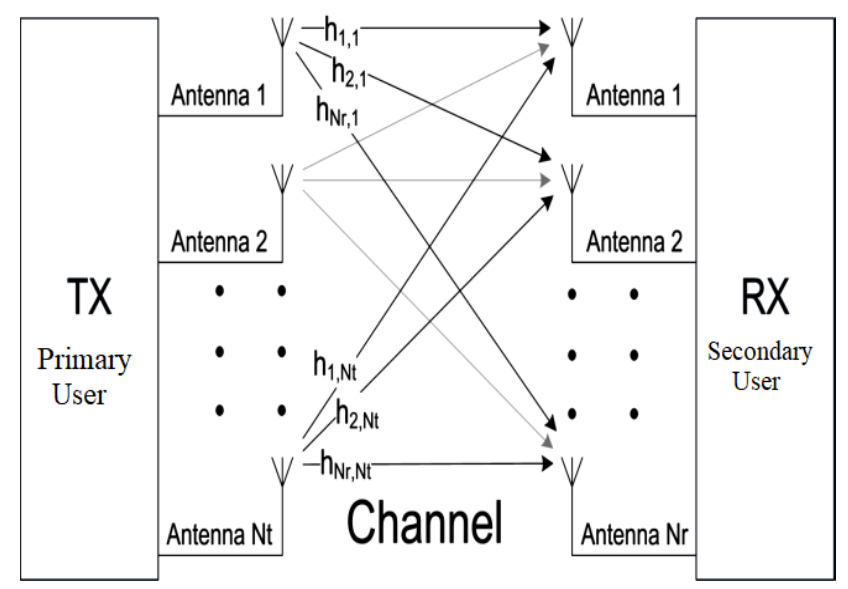

Fig. 1 MIMO communication system

As it is known, in addition to increasing the channel capacity, MIMO systems also reduce the negative effects of noise thanks to the spatial diversity of the antennas.

\section{Cognitive Radio And Basic Spectrum Sensing}

Suppose there is a Primary Base Station (PBS) with $N_{t}$ antenna and CR users within this coverage area. The purpose of CR users is to determine whether the PBS is active / passive. Thus, if PBS is passive, this communication band is used opportunistically by CR users. In this detection model, if the PBS has $N_{t}$ antenna and $M$ CR users, this system can be defined as a MIMO system[12].

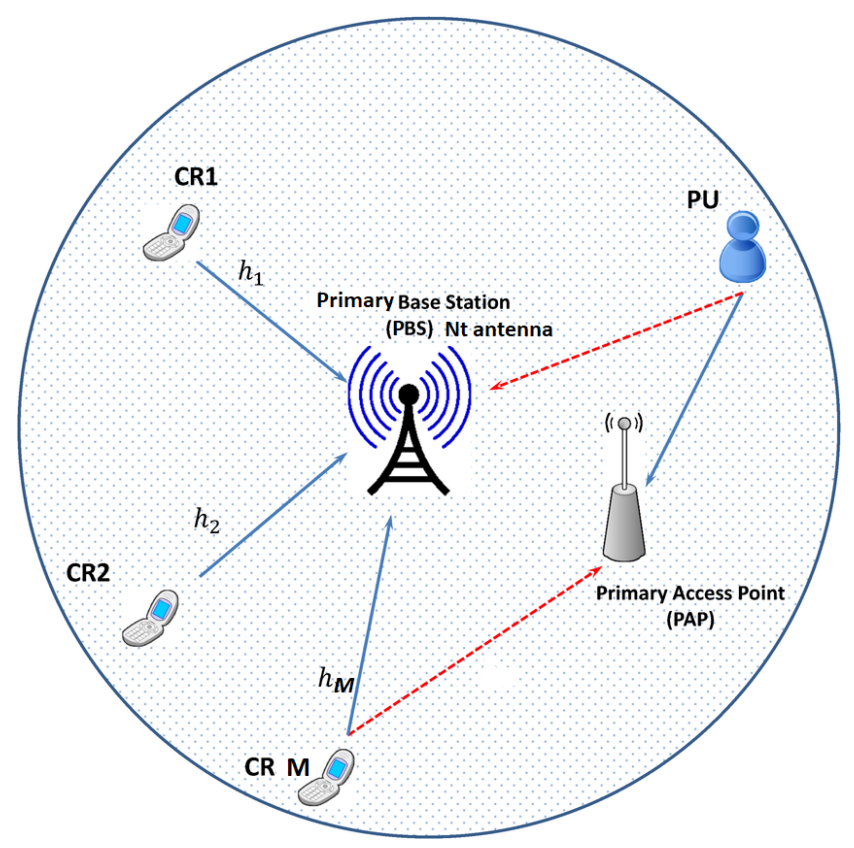

Fig. 2 Spectrum detection scenario for correlation based sensing

Basically the signal detection problem is to determine whether there is a embedded communication signal in the noise. In detection theory, this decision mechanism is explained by binary hypothesis testing. In binary hypothesis test, $H_{0}$ indicates that there is only a noise signal, $H_{1}$ indicates that it is a noise + communication signals. Mathematical decision making process is given below[13]

$$
\begin{gathered}
H_{0}: \boldsymbol{y}(k)=\boldsymbol{\eta}(k) k=1,2, \ldots .3, K \\
H_{1}: \boldsymbol{y}(k)=\boldsymbol{h}(k) \boldsymbol{s}(k)+\boldsymbol{\eta}(k) \quad k=1,2, \ldots .3, K
\end{gathered}
$$

Where $\boldsymbol{y}(k)$ is the form of the signal received from CR users (M numbers) stored in a matrix. For example, if $M$ is the number of CR users and the number of samples is $K$, the size of the $\boldsymbol{y}(k)$ matrix will be $M x K$. In addition to this where $\boldsymbol{\eta}(k)$ denotes the independent and identically distributed (i.i.d.) circularly symmetric Gaussian (CSCG) white noise. $\boldsymbol{h}(k)$ and $\boldsymbol{s}(k)$ represents channel coefficient vector and primary user signal, respectively. In multi-antenna BR systems, spectrum detection is given by comparing the test statistic that varies according to the method with the threshold. The spectrum detection model for CR systems is given below. 
BALKAN JOURNAL OF ELECTRICAL \& COMPUTER ENGINEERING, Vol. 9, No. 1, January 2021

$$
y_{m}(k)=\psi h_{m}(k) s_{m}(k)+\eta_{m}(k) m=1,2, \ldots .3, M
$$

Where $\psi=1$ under $H_{1}$ and $\psi=0$ under $H_{0}, M$ represents the number of $\mathrm{CR}$ user. In order to make a spectrum decision, the threshold and test statistics are compared in correlationbased spectrum detection. This phenome can be expressed mathematically as follows[12].

$$
T S_{\text {cor }} \underset{H_{0}}{\stackrel{H_{1}}{\gtrless}} \gamma_{\text {cor }} \quad H_{0} \text { or } H_{1}
$$

Where $T S_{\text {cor }}$ and $\gamma_{\text {cor }}$ define test statistics and threshold for correlation based detector, respectively.

\section{A. Correlation Based Spectrum Sensing}

Let $y_{m}(k)=s_{m}(k)+\eta_{m}(k)$ be the continuous-time received signal. Assume that we are interested in the frequency band with central frequency $W$ and bandwidth $f_{c}$. We sample the received signal at a sampling rate $f_{s}$. Thus, consecutive signal vectors for $y_{m}(k), s_{m}(k)$ and $\eta_{m}(k)$ are thus defined as follows.

$$
\begin{aligned}
& y_{m}(k)=\left[y_{m}(k) y_{m}(k-1) \ldots y_{m}(k-L+1)\right]^{T} \\
& s_{m}(k)=\left[s_{m}(k) s_{m}(k-1) \ldots s_{m}(k-L+1)\right]^{T} \\
& \eta_{m}(k)=\left[\eta_{m}(k) \eta_{m}(k-1) \ldots \eta_{m}(k-L+1)\right]^{T}
\end{aligned}
$$

Where $L$ is defined as the smoothing factor. Specifies how many samples the spectrum detection process will be done with. Thus, the statistical covariance matrices of the received signal $(\boldsymbol{y}(k))$ are defined as follows.

$$
\begin{aligned}
\mathbf{C}_{H_{0}} & =E\left[\boldsymbol{\eta}(k) \boldsymbol{\eta}^{\mathbf{T}}(k)\right] \text { under } H_{0} \\
\mathbf{C}_{H_{1}} & =E\left[\mathbf{y}(k) \mathbf{y}^{T}(k)\right] \text { under } H_{1}
\end{aligned}
$$

Equation 10 can be written as a result of Equation 10 and Equation 11.

$$
\mathbf{C}_{H_{0}}=\mathbf{C}_{H_{1}}+\sigma_{\eta}^{2} \mathbf{I}_{L}
$$

Where $\mathbf{I}_{L}$ denotes $L x L$ identity matrix. According to Equation 12 , if $y_{m}(k)=0, \quad \mathbf{C}_{H_{1}}=0$. Therefore, all non-diagonal components of $\mathbf{C}_{H_{0}}$ are equal to 0 . If there is a signal samples and the signal are correlated, $\mathbf{C}_{H_{1}}$ is not a diagonal matrix. Hence, some of the off-diagonal elements of $\mathbf{C}_{H_{0}}$ should be nonzeros. Denote $r_{m k}$ as the element of matrix $\mathbf{C}_{H_{0}}$ at the m.th row and $k$.th column. Then the following test statistics can be written.

$$
\begin{aligned}
& \delta_{1}=\frac{1}{L} \sum_{m=1}^{L} \sum_{k=1}^{L}\left|r_{m k}\right| \\
& \delta_{2}=\frac{1}{L} \sum_{m=1}^{L}\left|r_{m m}\right|
\end{aligned}
$$

At that time, under the $H_{0}, \delta_{1} /_{\delta_{2}}=1$. But under the $H_{0}$, $\delta_{1} \delta_{\delta_{2}}>1$. Consequently, ratio $\delta_{1} / \delta_{2}$ can be used to detect the presence of the signal.

\section{B. Determination threshold for Correlation based Spectrum Sensing}

To obtain the threshold value (under asymptotic conditions) the following equation can be written.

$$
\lim _{N \rightarrow \infty} E\left(\delta_{1}\right)=\sigma_{\eta}^{2}+\sigma_{s}^{2}+\frac{2 \sigma_{s}^{2}}{N} \sum_{l_{-} 1}^{L-1}(L-m)\left|\propto_{l}\right|
$$

Where $\sigma_{s}^{2}$ denotes variance of PBS. Under $H_{0}$;

$$
\begin{gathered}
\delta_{1 / \delta_{2}} \approx E\left(\delta_{1}\right) / E\left(\delta_{2}\right) \\
=1+(L-1) \sqrt{\frac{2}{\pi L}}
\end{gathered}
$$

As $L$ approaches infinity, this ratio approaches 1 . Thus, to obtain the threshold value, it should be continued with the conditional equation given below.

$$
\begin{aligned}
P_{f a} & =P\left(\delta_{1}<\gamma_{c o r} \delta_{2}\right) \\
& =P\left(\delta_{2}<\frac{1}{\gamma_{c o r}} \delta_{1}\right)
\end{aligned}
$$

If $\delta_{1}$ is written instead of Equation 19;

$$
\begin{aligned}
& \approx P\left(\delta_{2}<\frac{1}{\gamma_{c o r}} 1+(L-1) \sqrt{\frac{2}{\pi N}} \sigma_{\eta}^{2}\right) \\
& =P\left(\frac{\delta_{2}-\sigma_{\eta}^{2}}{\sqrt{\frac{2}{N}} \sigma_{\eta}^{2}}<\frac{\frac{1}{\gamma_{c o r}}\left(1+(L-1) \sqrt{\frac{2}{\pi N}}\right)-1}{\sqrt{\frac{2}{N}}}\right)
\end{aligned}
$$

Equation 21 rearranged;

$$
\approx 1-Q\left(\frac{\frac{1}{\gamma_{\operatorname{cor}}}\left(1+(M-1) \sqrt{\frac{2}{\pi N}}\right)-1}{\sqrt{\frac{2}{N}}}\right)
$$

Where

$$
Q(t)=\frac{1}{\sqrt{2 \pi}} \int_{t}^{\infty} e^{-u^{2} / 2} d u
$$


BALKAN JOURNAL OF ELECTRICAL \& COMPUTER ENGINEERING， Vol. 9, No. 1, January 2021

For a given $P_{f a}$, the associated threshold should be chosen such that[12];

$$
\gamma_{c o r}=\frac{1+(\mathrm{L}-1) \sqrt{\frac{2}{\mathrm{M} \pi}}}{1-\mathrm{Q}^{-1}\left(P_{f a}\right) \sqrt{\frac{2}{\mathrm{M}}}}
$$

According to Equation 24 the threshold value depends on the number of CR users, $L$ and $P_{f a} . P_{f a}$ is a value set by the Federal Communication Committee (FCC). For CR systems, this value is 0.1 .

\section{SIMULATION STUDIES}

In this section, we will give some simulation results for spectrum sensing performance and channel capacity. The simulation studies in this study were simulated in MATLAB environment. During the simulation studies, $P_{f a}$ is selected as 0,1 . Because this value is the limit value determined by the International Communication Committee. Additionally, noise uncertainty factor was not included in simulation studies. Because the Correlation Based Spectrum detection method is not affected by noise uncertainty factor[14], [15]. Looking at the threshold given with Equation 24, it is seen that there is no noise variance component. The absence of noise power when calculating the threshold indicates that this method is not affected by noise uncertainty.

In Fig. 2, only channel capacity is given for MIMO systems without detection performance. As can be seen, the highest channel capacity is reached when the antenna numbers is highest and the noise level is lowest. Although the increase in the antenna numbers in MIMO systems affects the channel capacity positively, it may not be easy to use more antennas. As explained in the previous chapters, the increase in the antenna numbers increases the spatial diversity, so the MIMO system is more resistant to noise.

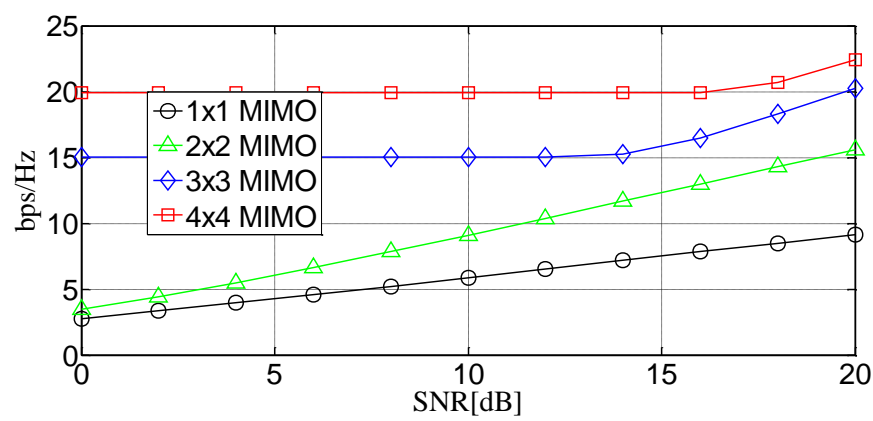

Fig. 3. Antenna versus channel capacity for MIMO syst

The antenna correlation should be at a minimum level in order to get maximum benefit from the spatial diversity. However, in many communication applications except CR applications, this may cause difficulties in practice, since the receiving antennas are located on the same receiver. In multi-user-single antenna CR applications, since each antenna is on a different user, maximum efficiency can be obtained from the spatial correlation.

One of the result graphics that constitute the main purpose of this study is given in Fig. 4. Where, the channel capacity for 4x4 and 6x6 MIMO systems is given along with the detection performance (the red numbers in the boxes describe the detection performance). Where $P_{d}=P\left(\delta_{1} /_{\delta_{2}}>\gamma_{\text {cor }}\right)$.

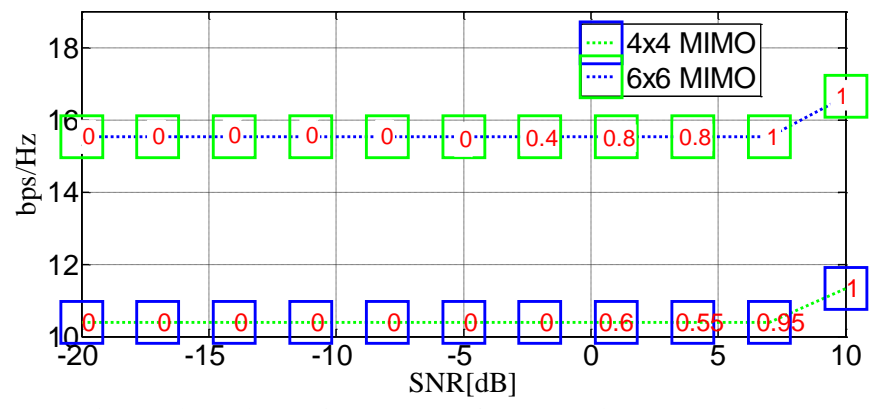

Fig. 4. Channel capacity versus $P_{d}$ for $4 \mathrm{X} 4$ and 6X6 MIMO systems

When calculating $P_{d}$, Monte Carlo analysis was applied. Thus, according to the detection scenario given in Figure 2, spectrum detection is performed by creating 500 times random PBS signal and channel coefficients. The average of 500 detection results is calculated for $P_{d}$. First of all, it is seen that the detection performance for the same noise levels in $4 \times 4$ and 6x6 MIMO systems is directly proportional to the antenna numbers. For example, in the presence of $1 \mathrm{~dB}$ noise, detection performances are measured as 0.95 and 1 for $4 \times 4$ and 6x6 MIMO. Moreover, at noise levels higher than $0 \mathrm{~dB}$, the $4 \times 4$ MIMO system has not made any accurate sensing at all. However, in a 6x6 MIMO system, it is seen that under $2 \mathrm{db}$ noise, 0.4 probability correct detection is made.

The antenna numbers and detection performance are given in Figure 5 for $2 \times 2$ and 3x3 MIMO systems. Unlike Figure 4, the most striking difference is that both channel capacity and detection performance are significantly reduced. For example, in the presence of $10 \mathrm{~dB}$ noise, the detection probability was 1 for 4x4 and 6x6 MIMO systems. However, in Figure 5, it is observed that these values decrease to 0,7 .

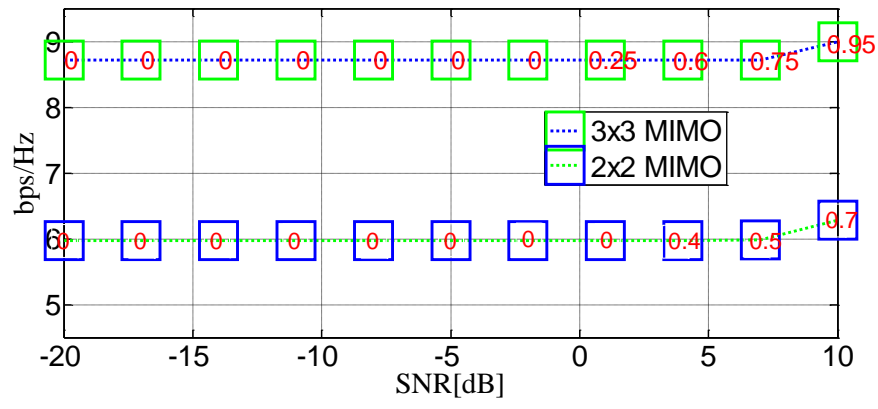

Fig. 5. Channel capacity versus $P_{d}$ for $3 \mathrm{X} 3$ and $2 \mathrm{X} 2 \mathrm{MIMO}$ systems

See Table $1 P_{d}$ versus SNR for different antenna numbers. $P_{d}$ and channel capacity are given separately for $5 \times 5$ and $8 \times 8$ MIMO systems that are not included in the graphs in Table 1 and Table 2.

TABLE I

SNR VERSUS $P_{d}$ FOR DIFFERENT MIMO SYSTEMS

\begin{tabular}{|c|c|c|c|c|}
\hline $\mathrm{SNR}$ & $4 \times 4$ & $5 \times 5$ & $6 \times 6$ & $8 \times 8$ \\
\hline $10 \mathrm{~dB}$ & 1 & 1 & 1 & 1 \\
\hline $5 \mathrm{~dB}$ & 0.55 & 0.7 & 0.8 & 0.93 \\
\hline $0 \mathrm{~dB}$ & 0.6 & 0.65 & 0.8 & 0.91 \\
\hline$-5 \mathrm{Db}$ & 0 & 0 & 0 & 0.2 \\
\hline
\end{tabular}


BALKAN JOURNAL OF ELECTRICAL \& COMPUTER ENGINEERING， Vol. 9, No. 1, January 2021

TABLE II

CHANNEL CAPACITY VERSUS SNR DIFFERENT MIMO SYSTEMS

\begin{tabular}{|c|c|c|c|c|}
\hline SNR & $4 \times 4$ & $5 \times 5$ & $6 \times 6$ & $8 \times 8$ \\
\hline $10 \mathrm{~dB}$ & 14 & 15 & 22 & 28 \\
\hline $5 \mathrm{~dB}$ & 11 & 13 & 16 & 18 \\
\hline $0 \mathrm{~dB}$ & 11 & 13 & 16 & 18 \\
\hline$-5 \mathrm{Db}$ & 11 & 13 & 16 & 18 \\
\hline
\end{tabular}

As can be seen from the tables, the $8 \times 8$ MIMO system is the most successful antenna combination in both channel capacity and detection performance.

\section{CONCLUSION}

In this study, correlation based spectrum sensing and channel capacity are investigated for MIMO systems. In the simulation studies, the effects of the antenna numbers on detection performance and channel capacity in MIMO systems containing different antenna numbers are examined. Thus, simulation studies were carried out by applying Monte Carlo analysis according to the given detection scenario. As a result of these simulation studies, the increase in the antenna numbers has positive effects on both detection performance and channel capacity.

\section{REFERENCES}

[1] B. B. Pradhan and L. P. Roy, "Ergodic capacity and symbol error rate of distributed massive MIMO systems over Rayleigh-inverse Gaussian fading channels using ZF detectors," Phys. Commun., vol. 38, no. 77, p. 7777, Feb. 2020, doi: 10.1016/J.PHYCOM.2019.100906.

[2] E. Dahlman, S. Parkvall, and J. Skold, 4G: LTE/LTE-Advanced for Mobile Broadband. 2013.

[3] M. Kang and M. S. Alouini, "Capacity of MIMO Rician channels," IEEE Trans. Wirel. Commun., vol. 5, no. 1, pp. 112-122, 2006, doi: 10.1109/TWC.2006.1576535.

[4] Y. Li, J. H. Winters, and N. R. Sollenberger, "MIMO-OFDM for wireless communications: Signal detection with enhanced channel estimation," IEEE Trans. Commun., vol. 50, no. 9, pp. 1471-1477, 2002, doi: 10.1109/TCOMM.2002.802566.

[5] M. Kowal, S. Kubal, P. Piotrowski, and R. Zieliński, "A simulation model of the radio frequency MIMO-OFDM system," Int. J. Electron. Telecommun., vol. 57, no. 3, pp. 323-328, 2011, doi: 10.2478/v10177-011-0043-6.

[6] C. Çiflikli and F. Y. Ilgin, "Covariance Based Spectrum Sensing with Studentized Extreme Eigenvalue," Tech. Gaz., vol. 25, no. 6, pp. 100-106, 2018.

[7] J. Wang, M. Ghosh, and K. Challapali, "Emerging cognitive radio applications: A survey," IEEE Commun. Mag., vol. 49, no. 3, pp. 74-81, 2011, doi: 10.1109/MCOM.2011.5723803.

[8] M. Matthaiou, M. R. McKay, P. J. Smith, and J. A. Nossek, "On the condition number distribution of complex wishart matrices," IEEE Trans. Commun., vol. 58, no. 6, pp. 1705-1717, 2010, doi: 10.1109/TCOMM.2010.06.090328.

[9] X. Liu, X. Zhang, H. Ding, and B. Peng, "Intelligent clustering cooperative spectrum sensing based on Bayesian learning for cognitive radio network," Ad Hoc Networks, vol. 94, 2019, doi: 10.1016/j.adhoc.2019.101968.

[10] P. D. Arapoglou, K. Liolis, M. Bertinelli, A. Panagopoulos, P. Cottis, and R. De Gaudenzi, "MIMO over satellite: A review," IEEE Commun. Surv. Tutorials, vol. 13, no. 1, pp. 27-51, 2011, doi: 10.1109/SURV.2011.033110.00072.

[11] N. Promsuwanna, P. Uthansakul, and M. Uthansakul, "Performance of antenna selection in MIMO system using channel reciprocity with measured data," Int. J. Antennas Propag., vol. 2011, 2011, doi: $10.1155 / 2011 / 854350$.
[12] Y. Zeng and Y. C. Liang, "Spectrum-sensing algorithms for cognitive radio based on statistical covariances," IEEE Trans. Veh. Technol., vol. 58, no. 4, pp. 1804-1815, 2009, doi: 10.1109/TVT.2008.2005267.

[13] R. A. Davis, O. Pfaffel, and R. Stelzer, "Limit theory for the largest eigenvalues of sample covariance matrices with heavy-tails," Stoch. Process. their Appl., vol. 124, no. 1, pp. 18-50, 2014, doi: 10.1016/j.spa.2013.07.005.

[14] Y. Zeng and Y. C. Liang, "Eigenvalue-based spectrum sensing algorithms for cognitive radio," IEEE Trans. Commun., vol. 57, no. 6, pp. 1784-1793, 2009, doi: 10.1109/TCOMM.2009.06.070402.

[15] Y. He, T. Ratnarajah, E. H. G. Yousif, J. Xue, and M. Sellathurai, "Performance analysis of multi-antenna GLRT-based spectrum sensing for cognitive radio," Signal Processing, vol. 120, pp. 580593, 2016, doi: 10.1016/j.sigpro.2015.10.018.

\section{BIOGRAPHIES}

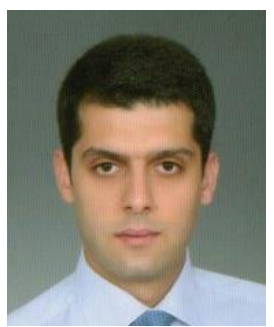

FATIH YAVUZ ILGIN is an Associate Professor in the Department of Electric and Electronics Engineering, Ezincan Binali YLDIRIM University, Turkey. Her research interests include: Statistical signal processing, Cognitive Radio systems, detection theory. 